\title{
Digital Necrosis in Type I Cryoglobulinemia
}

Sophie Georgin-lavialle ${ }^{1^{*}}$, Emmanuel Lafont ${ }^{2}$, Audrey Stansal $^{3}$ and Jacques Pouchot $^{2}$

${ }^{1}$ Department of Internal Medicine, Tenon Hospital, Pierre and Marie Curie University, France

${ }^{2}$ Department of Internal Medicine, Georges Pompidou European Hospital, Paris Descartes University, France

${ }^{3}$ Department of Vascular Medicine, Georges Pompidou European Hospital, Paris Descartes University, France

"Corresponding author: Sophie Georgin-lavialle, Department of Internal Medicine, Tenon hospital, Pierre and Marie Curie University, France, Tel: 33156017204; Fax: 33156017146; E-mail: sophie.georgin-lavialle@tnn.aphp.fr

Rec date: Nov 22, 2014, Acc date: Nov 24, 2014, Pub date: Nov 26, 2014

Copyright: () 2014 Faraj Sophie Georgin-lavialle et al. This is an open-access article distributed under the terms of the Creative Commons Attribution License, which permits unrestricted use, distribution, and reproduction in any medium, provided the original author and source are credited

\section{Abstract}

A 69-year-old man presented with severe pain in hands and feet related to necrosis. Extensive explorations revealed type I cyroglobulinemia associated to monoclonal IgG kappa. Unfortunately he died a few months after.

Keywords: Digital necrosis; Type I Cryoglobulinemia; IgG Kappa

\section{Clinical Image}

A 69-year-old man presented with severe pain in hands and feet. The distal part of most fingers and toes were necrotic (Figure 1). Extensive diagnostic work-up was performed: physical examination was otherwise unremarkable, and a thoracic and abdominal CT-scan was non-contributive. Blood sampling demonstrated the presence of a type I cryoglobulinemia, constituted of a monoclonal immunoglobulin $\mathrm{G}$ kappa at a titer of $2.03 \mathrm{~g} / \mathrm{L}$. Bone marrow smear and biopsy were normal. Treatment with oral corticosteroids and rituximab was instituted, and necrotic lesions were surgically excised. Unfortunately, the patient was readmitted one month later for extension of digital necrosis and infectious pneumonia. Outcome was unfavorable and the patient died. Type I cryoglobulinemia represent 10 to $15 \%$ of all cryoglobulinemia [1]. They are frequently associated with malignant B cell proliferation and the production of a precipitating monoclonal immunoglobulin that may result in severe ischemic manifestations such as the one reported here.

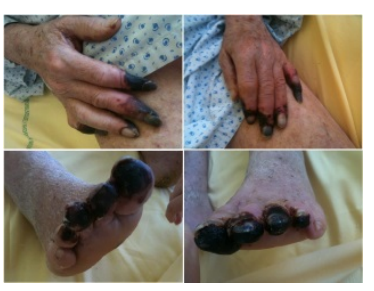

Figure 1: Distal part of toes and fingers were necrotic

\section{References}

1. Terrier B, Karras A, Kahn JE, Le Guenno G, Marie I, et al. (2013) The spectrum of type I cryoglobulinemia vasculitis: new insights based on 64 cases. Medicine (Baltimore) 92: 61-68. 